\title{
Lipopolysaccharide binding protein is an adipokine involved in the resilience of the mouse adipocyte to inflammation
}

\author{
José María Moreno-Navarrete ${ }^{1,2,3} \cdot$ Xavier Escoté $^{4,5,6} \cdot$ Francisco Ortega $^{1,2,3}$. \\ Marta Camps $^{7}$ - Wifredo Ricart ${ }^{1,2,3}$ - Antonio Zorzano ${ }^{6,7,8}$ - Joan Vendrell ${ }^{4,5,6}$. \\ Antonio Vidal-Puig ${ }^{9}$ • José Manuel Fernández-Real ${ }^{1,2,3}$
}

Received: 9 April 2015 / Accepted: 17 June 2015 / Published online: 23 July 2015

(C) Springer-Verlag Berlin Heidelberg 2015

\begin{abstract}
Aims/hypothesis Lipopolysaccharide (LPS) binding protein (LBP) is a novel $65 \mathrm{kDa}$ adipokine, linked to adipose tissue (AT) inflammation, obesity and insulin resistance, that inhibits adipocyte differentiation. Here, we investigated the molecular mechanisms behind these detrimental effects on adipogenesis through whole-genome transcriptomics and in vitro experiments.

Methods Permanent and transient knockdown (KD) and coculture experiments were performed in 3T3-L1 and 3T3F442A cell lines during adipocyte differentiation. Microarray gene expression was performed using Genechip Affymetrix technology and validated by real-time PCR.

Results LBP KD of 3T3-L1 cells led to a potentiated adipocyte differentiation with a dose-response relationship; genes involved in mitochondrial biogenesis, fatty acid metabolism and peroxisome proliferator-activated receptor $\gamma$ (PPAR- $\gamma$ )
\end{abstract}

action were dramatically upregulated in parallel to increased insulin signalling. Cells with LBP KD became refractory to proinflammatory cytokines and other inflammatory stimuli (LPS and palmitate). This phenotype, mediated through disrupted nuclear factor $\mathrm{\kappa B}(\mathrm{NF} \kappa \mathrm{B})$ signalling, was reversed by a soluble factor present in a co-culture with native cells and by exogenous LBP. Double-silencing of LBP and toll-like receptor 4 (TLR4) again rendered these cells insensitive to co-culture, LBP and inflammatory factors.

Conclusions/interpretation In summary, LBP is a proinflammatory soluble adipokine that acts as a brake for adipogenesis, strengthening the negative effects of palmitate and LPS on adipocyte differentiation.

Keywords Adipogenesis · Inflammation . Lipopolysaccharide binding protein $\cdot$ LPS · Palmitate $\cdot$ TLR4
Electronic supplementary material The online version of this article (doi:10.1007/s00125-015-3692-7) contains peer-reviewed but unedited supplementary material, which is available to authorised users.

José Manuel Fernández-Real

jmfreal@idibgi.org

1 Section of Diabetes, Endocrinology and Nutrition, Hospital of Girona 'Dr Josep Trueta', Carretera de França s/n, 17007 Girona, Spain

2 Institut d'Investigació Biomèdica de Girona (IdIBGi), Girona, Spain

3 Centro de Investigación Biomédica en Red de Fisiopatología de la Obesidad y Nutrición (CIBEROBN), Spain

http://www.ciberobn.es

4 Department of Endocrinology, Hospital Joan XXIII, Rovira i Virgili University, Tarragona, Spain
Institut d'Investigació Sanitaria Pere Virgili (IISPV), Tarragona, Spain

6 Centro de Investigación Biomédica en Red de Diabetes y Enfermedades Metabólicas Asociadas (CIBERDEM), Spain http://www.ciberdem.org

Departament de Bioquímica i Biologia Molecular, Facultat de Biologia, Universitat de Barcelona, Barcelona, Spain

8 Institute for Research in Biomedicine (IRB), Barcelona, Spain

9 Department of Clinical Biochemistry, Metabolic Research Laboratories, Institute of Metabolic Science, University of Cambridge, Addenbrooke's Hospital, Cambridge, UK 


$\begin{array}{ll}\text { Abbreviations } \\ \text { AT } & \text { Adipose tissue } \\ \text { KD } & \text { Knockdown } \\ \text { LBP } & \text { Lipopolysaccharide binding protein } \\ \text { LPS } & \text { Lipopolysaccharide } \\ \text { MCM } & \begin{array}{l}\text { LPS-stimulated macrophage-conditioned } \\ \text { medium }\end{array} \\ \text { NFkB } & \text { Nuclear factor kB } \\ \text { PPAR- } \gamma & \text { Peroxisome proliferator-activated receptor } \gamma \\ \text { sh(RNA) } & \text { Short hairpin (RNA) } \\ \text { sFA } & \text { Saturated fatty acids } \\ \text { si(RNA) } & \text { Small interfering (RNA) } \\ \text { siC } & \begin{array}{l}\text { Cells transfected with an empty vector but } \\ \text { otherwise able to synthesise LBP }\end{array} \\ \text { TLR4 } & \text { Toll-like receptor 4 }\end{array}$

\section{Introduction}

Adipose tissue (AT) dysfunction during the development of obesity is characterised by hypertrophy and hyperplasia of resident cells and enrichment of infiltrating inflammatory cells $[1,2]$. In a proinflammatory context, the normal function of AT depends on the resilience of adipocytes to the surrounding hostile cellular environment. Adipocyte-secreted factors are known to be important in the modulation of a variety of processes, including energy balance, adipogenesis and systemic inflammation. Lipopolysaccharide (LPS) binding protein (LBP) is a well-known liver acute-phase reactant that has recently been identified as an adipokine [3, 4]. The highest levels of LBP occur in fully differentiated human adipocytes from obese individuals [5]. Adipocyte LBP biosynthesis is associated with AT dysfunction and inflammation [5]. We found that levels of $L B P$ expression were low when AT was physiologically functional and adipocyte differentiation was viable in an insulin-sensitive environment [5]. Conversely, LBP levels were increased under conditions of impaired adipose differentiation in association with insulin resistance [3, 5, 6]. However, these previous correlative studies do not allow a causative link to be defined for LBP and these two variables. In the current study, we aimed to investigate in depth the role of LBP in adipocyte dysfunction through whole-genome transcriptomics and in vitro experiments in 3T3-L1 and 3T3F442A cell lines.

\section{Methods}

3T3-L1 and 3T3-F442A adipocyte differentiation Embryonic fibroblast mouse 3T3-L1 cells (American Type Culture Collection; LGC Standards, Barcelona, Spain) were maintained in DMEM containing $20 \mathrm{mmol} / 1$ glucose, $10 \%$ (vol./vol.) FBS, $100 \mathrm{U} / \mathrm{ml}$ penicillin and $100 \mu \mathrm{g} / \mathrm{ml}$ streptomycin. At 2 days after confluence, insulin $(5 \mu \mathrm{g} / \mathrm{ml})$, dexamethasone $(0.5 \mu \mathrm{mol} / \mathrm{l})$ and isobutylmethylxanthine $(0.5 \mathrm{mmol} / \mathrm{l})$ were added for 2 days, followed by insulin $(5 \mu \mathrm{g} / \mathrm{ml})$ alone for 5 days. The medium for 3T3-F442A cell growth was DMEM supplemented with $10 \%$ FBS, $100 \mathrm{U} / \mathrm{ml}$ penicillin and $100 \mu \mathrm{g} / \mathrm{ml}$ streptomycin in a humidified atmosphere of $5 \% \mathrm{CO}_{2}$ at $37^{\circ} \mathrm{C}$. Cells were grown to confluence and, after 2 days, differentiation was induced by switching cells to DMEM, 10\% FBS and $50 \mathrm{ng} / \mathrm{ml}$ insulin. Differentiation was monitored by morphological assessment and adipogenic gene expression.

During 3T3-L1 differentiation, LPS $(0.1$ and $1 \mu \mathrm{g} / \mathrm{ml})$, palmitate (100 and $250 \mu \mathrm{mol} / \mathrm{l})$ and LPS-stimulated macrophageconditioned medium (MCM) [0.25\% and 2.5\% (vol./vol.)] were added. Palmitate experimental procedures were performed as described previously [7]. Briefly, palmitate stock solution was dissolved in PBS-BSA $(3.3 \mathrm{mmol} / \mathrm{l})$ solution, which was used as a vehicle. MCM was obtained from THP-1 cells previously exposed to $10 \mathrm{ng} / \mathrm{ml}$ LPS in RPMI1640 serum-free medium for $24 \mathrm{~h}$ in a humidified atmosphere of $5 \% \mathrm{CO}_{2}$ at $37^{\circ} \mathrm{C}$. Rosiglitazone $(1 \mu \mathrm{mol} / \mathrm{l})$ was added during 3T3-F442A differentiation.

Short hairpin and small interfering RNA knockdown We applied short hairpin (sh)RNA (Sigma MissionRNAi) to stably knock down LBP and gene expression in 3T3-L1 fibroblasts (SHCLNV-NM_008489; Sigma, Barcelona, Spain). We used five plasmids containing mouse-Lbp-targeting small interfering (si)RNAs cloned into pLKO.1-puro shRNA vector and a non-targeting shRNA control plasmid.

In order to produce lentivirus, plasmid DNAs were transfected into subconfluent HEK 293T cells together with $\mathrm{pCMV} \Delta 8,74$ helper packaging construct and $\mathrm{pMD} 2 \mathrm{G}$ vector encoding envelope protein (kindly provided by $\mathrm{D}$. Trono from the Ecole Polytechnique Federale de Lausanne, Lausanne, Switzerland). Culture medium containing lentivirus was harvested $48 \mathrm{~h}$ and $72 \mathrm{~h}$ after transfection and passed through $0.4 \mu \mathrm{m}$ cellulose acetate filters. Recombinant lentivirus was concentrated and purified on a $20 \%$ (wt/vol.) sucrose cushion by ultracentrifugation $(2 \mathrm{~h}$ at $46,000 \mathrm{~g}$ ).

Toll-like receptor 4 (TLR4) knockdown in LBP-depleted 3T3-L1 was performed using Tlr4-targeted and control shRNA lentiviral particles (sc-40260-V and sc-108080; Santa Cruz Biotechnology, CA, USA). Positive 3T3-L1 fibroblasts harbouring the shRNA cassette for LBP were selected by puromycin selection $60 \mathrm{~h}$ after infection.

For the siRNA experiments, electroporation of 3T3-F442A was conducted in the pre-adipocyte phase. Cells were suspended by mild trypsinisation and electroporated using the Gene Pulser Electroporator (Bio-Rad Laboratories, Madrid, Spain), according to the manufacturer's recommendations. Briefly, about 2 million cells were collected and resuspended in $200 \mu$ l electroporation buffer (Bio-Rad Laboratories). For the siRNA experiments, control (D-001810-01-05; 
Dharmacon, CULTEK, Madrid, Spain) or mice $L b p$ (L-047095-01-0005; Dharmacon) siRNAs were delivered into pre-adipocytes $(250 \mathrm{nmol} / \mathrm{l})$. Pre-adipocytes were then reseeded onto 12-well plates.

Co-culture experiments Co-culture of si $L b p-3 \mathrm{~T} 3-\mathrm{L} 1$, siLbpsiTlr4-3T3-L1 and 3T3-L1 cells transfected with an empty vector ( $\mathrm{siC}-3 \mathrm{~T} 3-\mathrm{L} 1$ cells) was performed using Corning Transwell polyester membrane cell culture inserts (24 mm Transwell with $0.4 \mu \mathrm{m}$ pore polyester membrane insert [VWR International Eurolab, Barcelona, Spain]) throughout cell differentiation. The comparator was a co-culture of siLbp- and siLbp-siTlr4-3T3-L1 cells at both sides of the Transwell.

RNA purification and gene expression analysis RNA was prepared from these samples using RNeasy Lipid Tissue Mini Kit (QIAGEN, Izasa, Barcelona, Spain). The integrity of each RNA sample was checked by Agilent Bioanalyzer (Agilent Technologies, Palo Alto, CA, USA). Total RNA was quantified using a spectrophotometer (GeneQuant, GE Health Care, Piscataway NJ, USA) and reverse transcribed to cDNA using the High Capacity cDNA Archive Kit (Applied Biosystems, Madrid, Spain) according to the manufacturer's protocol.

Gene expression was assessed by real-time PCR using a LightCycler 480 Real-Time PCR System (Roche Diagnostics, Barcelona, Spain); TaqMan and SYBR Green technology was used to quantify the relative genetic expression. The commercially available and pre-validated TaqMan primer/probe sets used were as follows: endogenous control $18 \mathrm{~S}$ and target gene mouse Lbp (Mm00493139_m1), Fasn (Mm00662319_m1), Pparg (Mm00440940_m1), Adipoq (Mm00456425_m1), Fabp4 (Mm00445880_m1), Cebpa (Mm00514283_s1), Tlr4 (Mm00445273_m1), Slc2a4 (also known as Glut4) (Mm01245502_m1), Dlk (also known as Pref1) (Mm00494477_m1) (Applied Biosystems). Il6 and Mcp1 (also known as $C c l 2$ ) were assessed by real-time PCR using SYBR Green technology suitable for relative genetic expression quantification. The RT-PCR TaqMan and SYBR Green reaction was performed in a final volume of $25 \mu$ l. The cycle comprised initial denaturing for $10 \mathrm{~min}$ at $95^{\circ} \mathrm{C}$, then 40 cycles of a $15 \mathrm{~s}$ denaturising phase at $95^{\circ} \mathrm{C} / 1 \mathrm{~min}$ annealing and extension phase at $60^{\circ} \mathrm{C}$. A threshold cycle $\left(\mathrm{C}_{\mathrm{t}}\right.$ value $)$ was obtained for each amplification curve and a $\Delta \mathrm{C}_{\mathrm{t}}$ value was calculated by subtracting the $\mathrm{C}_{\mathrm{t}}$ value for $18 \mathrm{~S}$ RNA from the $\mathrm{C}_{\mathrm{t}}$ value for each sample. Fold changes compared with the endogenous control were then determined by calculating $2^{-\Delta C_{t}}$, so gene expression results are expressed as expression ratio relative to $18 \mathrm{~S}$ gene expression according to manufacturers' guidelines.
Microarray gene expression Gene expression analysis was performed using Genechip Affymetrix technology. All procedures (sample processing, hybridisation, developing, chip scanning and result analyses) were performed following manufacturers' protocols for the equipment (Affymetrix, Santa Clara, CA, USA), and using Mouse Gene 1.0 ST Array, Ambion WT Expression and Genechip WT Terminal Labeling kits. The siLBP-3T3-L1 cells were compared with siC3T3-L1 cells at day 7 of adipocyte differentiation. Microarray analysis was performed in three independent experiments. Two types of software were used to analyse the results: Command Console (AGCC 3.2.4) and Expression Console (EC 1.1), both from Affymetrix.

Secreted LBP measurement Levels of LBP in adipocyteconditioned medium were measured using the mouse LBP ELISA kit (Hycult Biotech, PB Uden, the Netherlands) according to the manufacturer's instructions. Intra- and inter-assay coefficients of variation for these determinations were between $5 \%$ and $10 \%$. The level of detection was $4.4 \mathrm{ng} / \mathrm{ml}$.

Endotoxin (LPS) and NEFA levels in cell culture medium were measured using the Limulus Amebocyte Lysate test (QCL-1000; Lonza Iberica, Barcelona, Spain) and Free Fatty Acid Quantification Kit (K612-100; BioVision, Milpitas, CA, USA), as recommended by the manufacturer.

${ }^{\text {S473 Akt, }}{ }^{(\text {panY) }}$ IRS, ${ }^{\text {S536 }}$ NF KB, ${ }^{\text {T183/Y185 JNK, T180/ }}$ ${ }^{\text {Y182 }}$ p38MAPK phosphorylation measurement by ELISA The PathScan sandwich ELISA kit for ${ }^{\mathrm{p}-\mathrm{S} 473} \mathrm{Akt}$, total $^{A k t,}{ }^{p-(p a n Y)}$ IRS, ${ }^{p-S 536}$ nuclear factor $\kappa$ B (NFkB), total NFkB, ${ }^{\mathrm{p}-\mathrm{T} 183 / \mathrm{Y} 185}$ mitogen-activated protein kinase 8 (JNK), ${ }^{\mathrm{p}-\mathrm{T} 180 / \mathrm{Y} 182}$ p38 map kinase (p38MAPK) comprises seven solid-phase sandwich ELISAs that detect endogenous levels of named intracellular proteins. Antibodies to ${ }^{\mathrm{p}-\mathrm{S} 473} \mathrm{Akt}$, total Akt, ${ }^{\mathrm{p}-(\mathrm{panY})}$ IRS, ${ }^{\mathrm{p}-\mathrm{S} 536}{ }^{\mathrm{NF}} \mathrm{KB}$, total NFkB, ${ }^{\mathrm{p}-\mathrm{T} 183 / \mathrm{Y} 185} \mathrm{JNK}$, and ${ }^{\mathrm{p}-\mathrm{T} 180 / \mathrm{Y} 182}$ p38MAPK are coated onto the microwells. After incubation with cell lysates, the proteins are captured by the coated antibody. Following extensive washing, the corresponding mouse monoclonal antibody is added to detect the captured protein in each ELISA. Horseradish peroxidase (HRP)-linked anti-mouse antibody recognises the bound detection antibody. HRP substrate, tetramethylbenzidine (TMB), is added to develop colour. The magnitude of optical density for this developed colour is proportional to the quantity of ${ }^{\mathrm{p}-\mathrm{S} 473} \mathrm{Akt}$, total Akt, ${ }^{\mathrm{p}-(\mathrm{panY})}$ IRS, ${ }^{\mathrm{p}-\mathrm{S} 536} \mathrm{NF} \times \mathrm{B}$, total NFKB, ${ }^{\mathrm{p}-\mathrm{T} 183 / \mathrm{Y} 185} \mathrm{JNK}$ and $\mathrm{p}-\mathrm{T} 180 / \mathrm{Y} 182 \mathrm{p} 38 \mathrm{MAPK}$ protein. Specificity and sensitivity tests were performed by the manufacturers using western blot analysis. Intra- and inter-assay coefficients of variation for all these determinations were between $5 \%$ and $10 \%$.

To evaluate the effects of LBP KD on insulin signalling during pre-adipocyte differentiation, we investigated ${ }^{\mathrm{S} 473} \mathrm{Akt}$ 
and ${ }^{\left(p^{2} Y\right)}$ IRS1 phosphorylation under insulin stimulation (100 nmol/l) for $10 \mathrm{~min}$ at the end of 3T3-L1 cell differentiation (day 7).

Statistical methods Unless stated otherwise, statistical analyses were performed using SPSS statistical software (SPSS v13.0; IBM, Chicago, IL, USA). The non-parametric MannWhitney test was used. Levels of statistical significance were set at $p<0.05$. For microarray gene expression bioinformatic analyses, the software Partek Genomics Suite (Partek Incorporated, St. Louis, MO, USA) was used. ANOVA, Bonferroni and false discovery rate (FDR) multiple correction tests were used for statistical analysis.

\section{Results}

LBP KD facilitates adipocyte differentiation and insulin signalling and prevents inflammatory changes Remarkably, LBP KD led to enhanced adipocyte differentiation and fat storage even when these cells were challenged with an inflammatory environment. Differentiation was accelerated in a dose-response silenced-dependent manner (in 3T3-L1 cells (Fig. 1a, b) and in 3T3-F442A (electronic supplementary material [ESM] Fig. 1)). Adipogenesis induced with rosiglitazone, $1 \mu \mathrm{mol} / 1$, decreased $L b p$ gene expression in 3T3-F442A cells (ESM Fig. 1). This finding is similar to findings in human adipocytes and 3T3-L1 cells [5]. The LBP KD cells were characterised by an accelerated $\mathbf{a}$

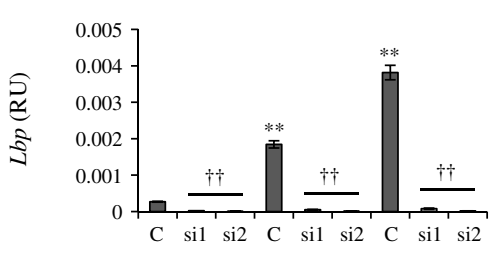

b

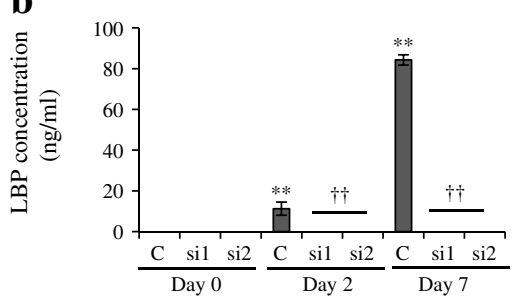

c

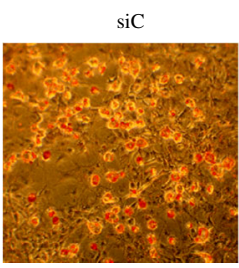

d

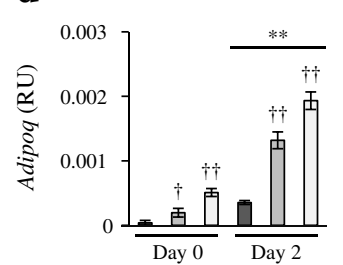

h

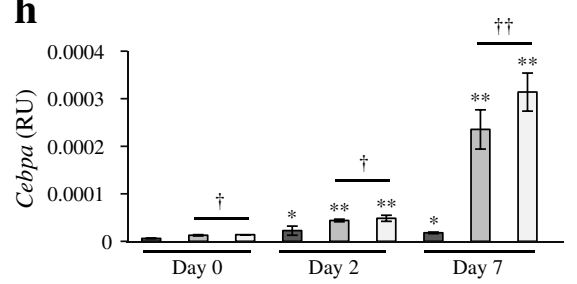

$\mathbf{j}$

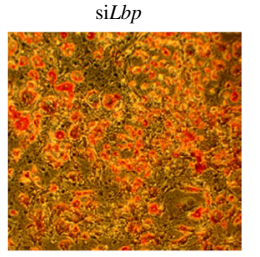

l

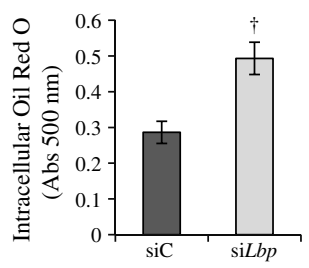

$\mathbf{e}$
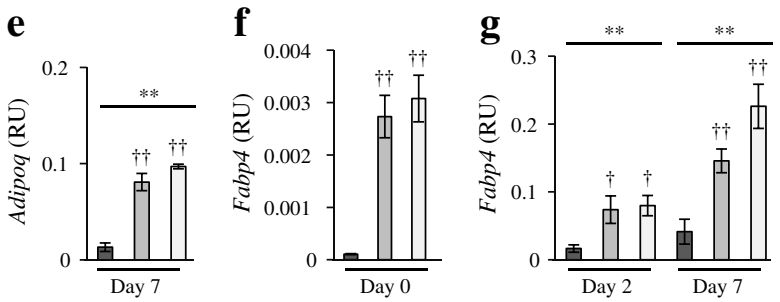

i

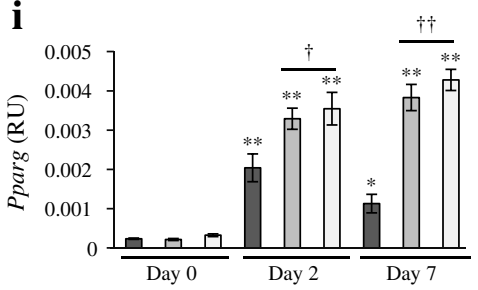

$\mathbf{k}$

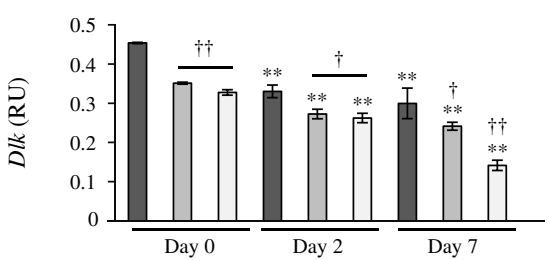

m

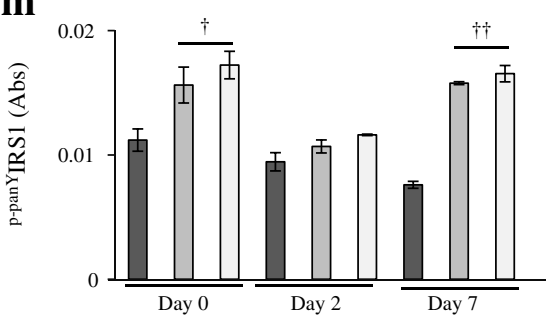

Fig. 1 (a, b) $L b p$ gene expression (a) and LBP protein secretion (b) in 3T3-L1 $L b p$-KD cells (silenced with siRNA1 and siRNA2) during 3T3L1 differentiation at days 0,2 and 7. (c) Microscopy image $(\times 10$ magnification) from negative $\mathrm{siC}$ and siLbp at day 7 of 3T3-L1 differentiation with Oil Red $\mathrm{O}$ staining. (d-m) The effect of LBP KD (siRNA1 and siRNA2) on Adipoq (d, e), Fabp4 (f, g), Cebpa (h), Pparg (i), Fasn (j) and $D l k(\mathbf{k})$ gene expression and insulin-stimulated ${ }^{\mathrm{p}-\mathrm{S} 473}$ Akt (l) and

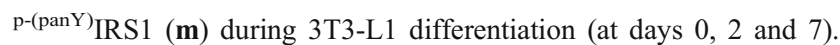
${ }^{*} p<0.05$ and $* * p<0.01$ compared with day $0 ;{ }^{\dagger} p<0.05$ and ${ }^{\dagger \dagger} p<0.01$ compared with siC. Dark grey bars, siRNA negative or siC; light grey bars, siRNA1 $L B P$ with $62 \%$ depletion ( $\operatorname{si} L b p$ or si1,); white bars, siRNA2 $L b p$ with $92 \%$ of depletion (si2). All experiments were performed in triplicate. Data are represented as mean \pm SEM. C, siC; RU, relative units 
accumulation of lipid droplets (Fig. 1c). Whole-genome microarray gene expression analysis revealed that most of the genes upregulated in siLbp-3T3-L1 vs siC-3T3-L1 cells were linked to adipocyte differentiation and adipocyte-associated metabolic processes (see ESM Table 1). Most of the biological processes and metabolic pathways potentiated by LBP KD were linked to adipocyte metabolism and adipogenesis (mitochondrial biogenesis [such as citrate cycle, fatty acid elongation in mitochondria, oxidative phosphorylation and pyruvate metabolism], fatty acid metabolism and peroxisome proliferator-activated receptor $\gamma$ [PPAR- $\gamma]$ signalling; adjusted Bonferroni $p<0.00001$, ESM Fig. 2a). Real-time PCR confirmed a significant increase in the expression of lipogenic and adipogenic genes (Fasn, Pparg, Adipoq, Fabp 4 and Cebpa) (Fig. 1d-j), decreased expression of Dlk (a negative marker of adipogenesis [8]) (Fig. 1k) and increased activity of the insulin-signalling pathway (Akt phosphorylation and IRS1 tyrosine phosphorylation) after insulin stimulation (100 nmol/1, $10 \mathrm{~min})$ in the KD cells (Fig. 11-m).

Levels of ${ }^{\mathrm{p}-\mathrm{S} 536} \mathrm{NF} \kappa \mathrm{B}$, used as a marker of NFKB activity, were significantly decreased in si $L b p-3 \mathrm{~T} 3-\mathrm{L} 1$ vs siC-3T3-L1

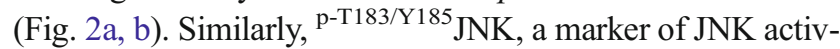
ity linked to stress pathways negatively associated with adipogenesis, also tended to be decreased in siLbp-3T3-L1 cells

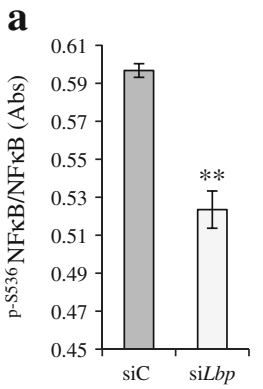

b

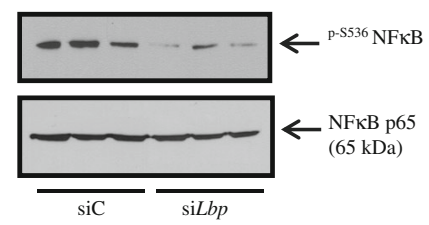

$\mathbf{e}$

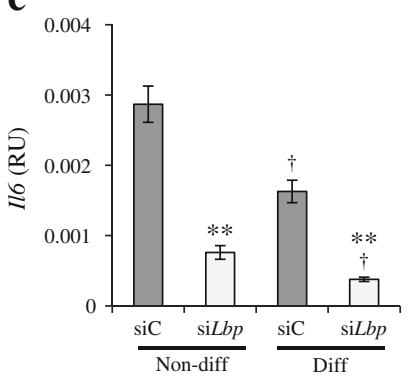

f

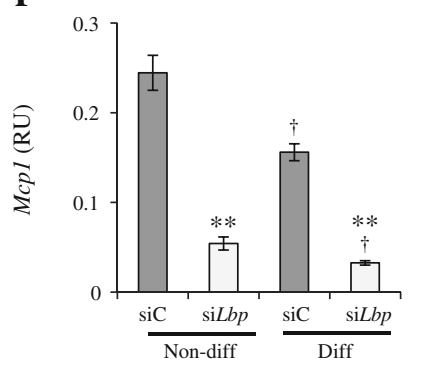

g

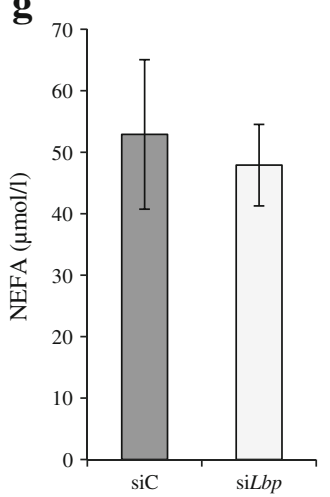

c

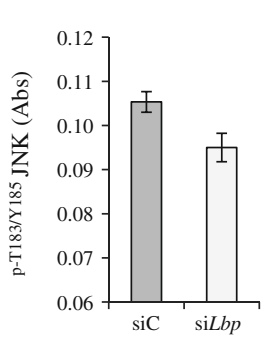

d

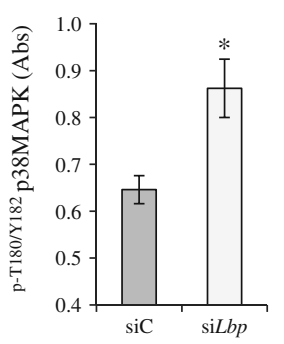

h

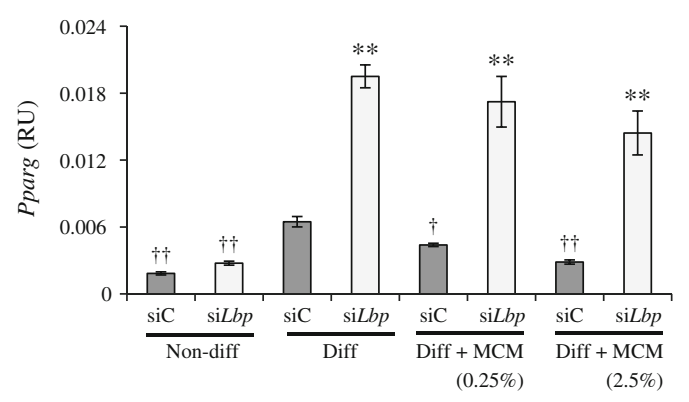

i

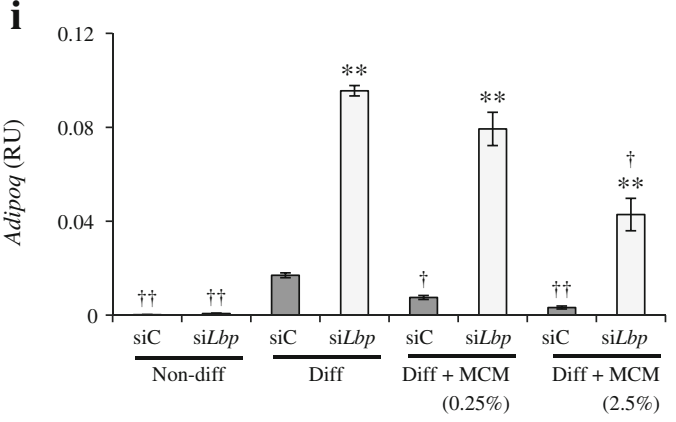

differentiated. (g) The effects of LBP KD (siLbp) on NEFA levels in cell culture medium at day 7. (h, i) The effects of LPS-stimulated MCM $(0.25 \%$ and $2.5 \%)$ on expression of the adipogenic genes Pparg (h) and Adipoq (i) during adipocyte differentiation in $\mathrm{SiC}$ and siLbp cells at day 7 . ${ }^{* *} p<0.01$ compared with siC. ${ }^{\dagger} p<0.05$ and ${ }^{\dagger \dagger} p<0.01$ compared with differentiated. Dark grey bars, siC; light grey bars, siL $b p$. All experiments were performed in triplicate. Data are represented as mean \pm SEM. Diff, differentiated; RU, relative units 
(Fig. 2c). However, ${ }^{\mathrm{p}-\mathrm{T} 180 / \mathrm{Y} 182}$ p38MAPK, known to be increased with adipocyte differentiation, was increased in siLbp3T3-L1 cells (Fig. 2d).

In parallel with accelerated adipogenesis and increased insulin signalling, LBP KD led to decreased expression of inflammation-associated genes (Il6 and Mcpl) (Fig. 2e, f). While LPS was not detected, substantial amounts of NEFA were detectable in the cell culture medium at day 7 . However, LBP KD had no significant effects on NEFA levels (Fig. 2g). Furthermore, LBP KD attenuated the anti-adipogenic effects (specifically the decrease in Pparg and Adipoq gene expression) of exposure to MCM $(0.25 \%$ and $2.5 \%)$ (i.e. inflammation-like conditions) (Fig. $2 \mathrm{~h}-\mathrm{i}$ ). Similarly, the negative effects of LPS $(0.1$ and $1 \mu \mathrm{g} / \mathrm{ml})$ and palmitate (100 and $250 \mu \mathrm{mol} / \mathrm{l})$ administration on adipocyte differentiation, decreasing expression of adipogenic genes (Adipoq, Pparg and
Slc2a4 [9]) and inducing the inflammatory pathway (Il6 gene expression and ${ }^{\mathrm{p}-\mathrm{S} 536} \mathrm{NF} \mathrm{KB}$ ) were absolutely attenuated in LBP KD cells (Fig. 3a-e). In contrast, ${ }^{\mathrm{p}-\mathrm{T} 183 / \mathrm{Y} 185}$ JNK levels and ${ }^{\mathrm{p}-\mathrm{T} 180 / \mathrm{Y} 182}$ p38MAPK levels remained unchanged in all circumstances (Fig. 3f, g).

Rescue experiment with exogenous LBP reversed the proadipogenic effects of LBP KD Addition of exogenous LBP $(10 \mathrm{ng} / \mathrm{ml})$ to LBP KD and control cells decreased the expression of adipogenic genes at days 2 (Fig. 4a-f) and 7 (Fig. 4g-1). The dependency of these effects on the secreted LBP was further confirmed by co-culturing siLbp-3T3-L1 cells with cells transfected with an empty vector but otherwise able to synthesise LBP (siC-3T3-L1 cells) in a Transwell co-culture system (Fig. 5a). This led to a reversal of the phenotype of the LBP KD cells, with blunted adipogenesis and lipogenesis
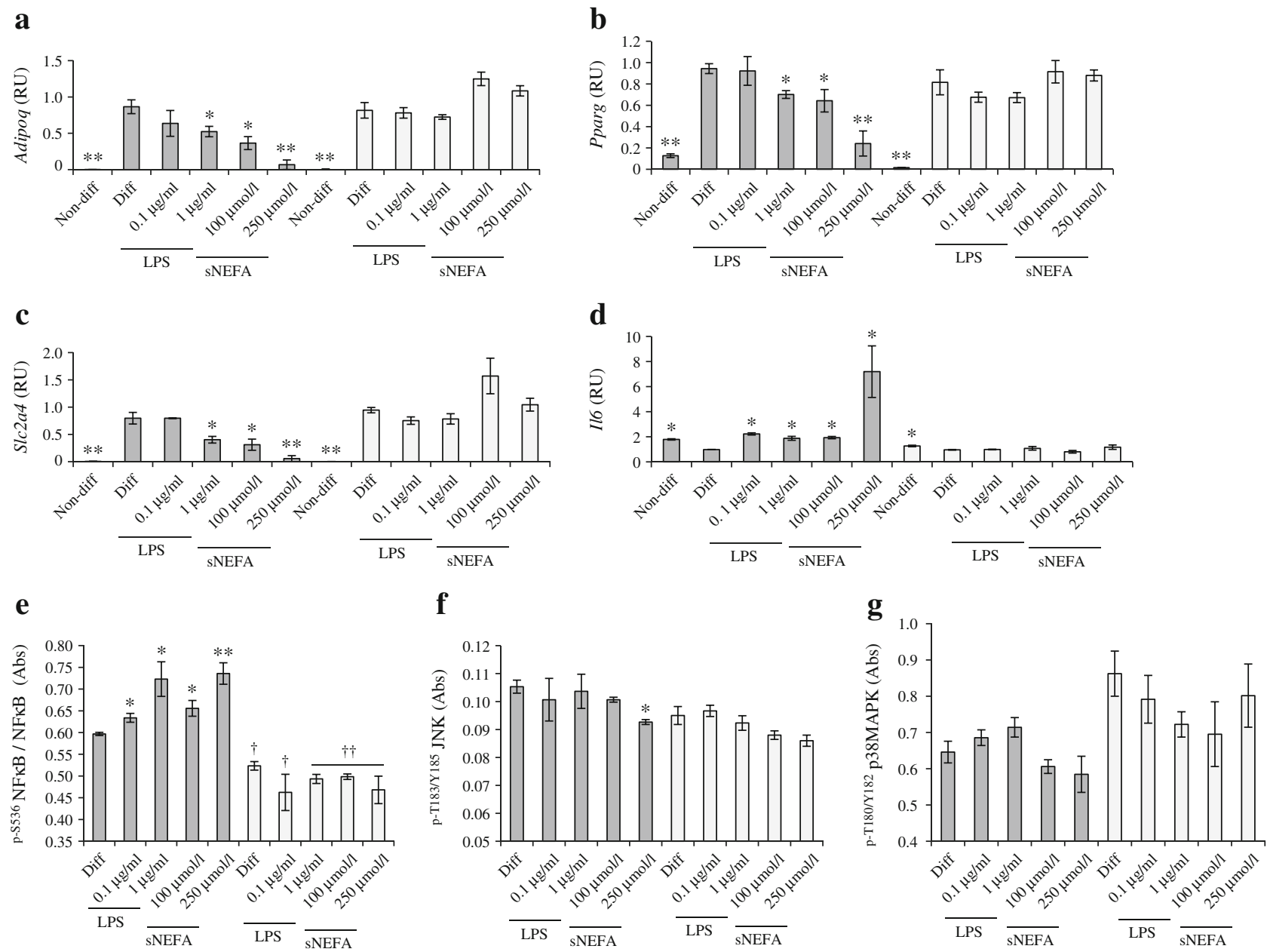

Fig. $3(\mathbf{a}-\mathbf{g})$ The effects of LPS $(0.1$ and $1 \mu \mathrm{g} / \mathrm{ml})$ and saturated NEFA (palmitate, 100 and $250 \mu \mathrm{mol} / \mathrm{l}$ ) on Adipoq (a), Pparg (b), Slc2a4 (c) and Il6 (d) levels and on NFKB (e), JNK (f) and p38MAPK (g) activities in $s i C$ and si $L b p$ during adipocyte differentiation at day 7 . The effect on protein activity was calculated indirectly from the degree of ${ }^{\mathrm{p}-\mathrm{S} 536} \mathrm{NFKB}$, ${ }^{\mathrm{p}-\mathrm{T} 183 / \mathrm{Y} 185} \mathrm{JNK}$, $\mathrm{p}$-T180/Y182 $\mathrm{p} 38 \mathrm{MAPK}$ (higher phosphorylation=more activity). As this figure

shows the effects of LPS and saturated NEFA according to LBP KD, gene expression values $(\mathbf{a}-\mathbf{d})$ are normalised to the mean value of differentiation control for each cell type (siC or siLbp). ${ }^{*} p<0.05$ and ${ }^{* *} p<0.01$ compared with the differentiation control; ${ }^{\dagger} p<0.05$ and ${ }^{\dagger \dagger} p<0.01$ compared with siC. Dark grey bars, siC; light grey bars, si $L b p$. All experiments were performed in triplicate. Data are represented as mean \pm SEM. Diff, differentiated; RU, relative units; sNEFA, saturated NEFA 
(Fig. 5b-f), increased inflammatory markers (Fig. 5g) and decreased intracellular lipid accumulation (ESM Fig. 3a). The LBP concentration in the media of cells transfected with an empty vector was tenfold higher vs siLbp-3T3-L1 cells (38.6 $\pm 3.91 \mathrm{vs} 3.2 \pm 1.18 \mathrm{ng} / \mathrm{ml}$ ) (Fig. $5 \mathrm{~h}$ ).

Effects of TLR4 knockdown on LBP-mediated effects It is well known that the activation of ${ }^{\mathrm{p}-\mathrm{S} 536} \mathrm{NF} \kappa \mathrm{B}$ on LPS and palmitate is induced through the TLR4 pathway, and that LBP enhancement of LPS inflammatory effects in macrophages requires TLR4. We investigated the requirement for TLR4 to mediate the anti-adipogenic effects of LBP by performing a double-silencing of LBP and TLR4 (TLR4LBP KD) using shRNA lentiviral transfection to decrease levels of $T l r 4$ by $\sim 35 \%$ (Fig. $6 a$ ) and $L b p$ by $\sim 90 \%$ (Fig. 6b). The TLR4-LBP KD cells tended to differentiate better than LBP KD cells (Fig. $6 \mathrm{c}-\mathrm{e}$ ). In fact, the fold increase in adipogenic genes in TLR4-LBP KD cells tended to be increased in comparison with LBP KD cells when analysed by microarray (see ESM Table 2). Again, most of the biological processes and metabolic pathways that were significantly potentiated (Bonferroni adjusted $p<0.00001$ ) were related to adipocyte metabolism and adipogenesis (ESM Fig. 2b). As for LBP KD, no significant effects of TLR4-LBP KD on NEFA levels were observed (ESM Fig. 3b). No significant effects on

Fig. 4 (a-l) The effects of LBP $(10 \mathrm{ng} / \mathrm{ml})$ administration on the expression of adipogenic gene markers in si $L b p$ - and siC-3T3-L1 during adipocyte differentiation at day $2(\mathbf{a}-\mathbf{f})$ and at day $7(\mathbf{g}-\mathbf{l})$. $* p<0.05$ and $* * p<0.01$ compared with vehicle control; ${ }^{\dagger} p<0.05$ and ${ }^{\dagger \dagger} p<0.01$ compared with siC. Dark grey bars, siC; light grey bars, siLbp. All experiments were performed in triplicate. Data are represented as mean \pm SEM. C, control; RU, relative units
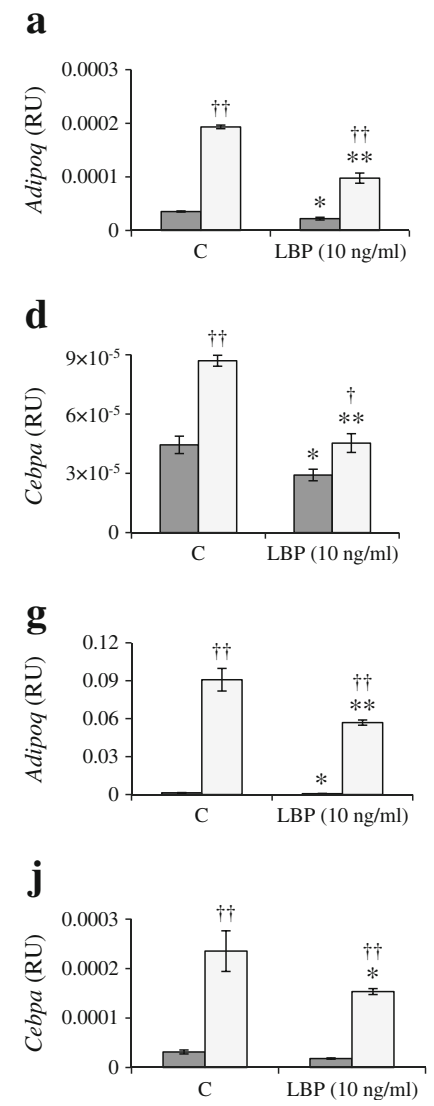

Tlr4 gene expression were observed in LBP KD cells at day 7 (Fig. 6a).

Co-culture of TLR4-LBP KD cells with cells transfected with an empty vector (siC-3T3-L1 cells) in a Transwell coculture system (Fig. 7a, b) showed, in sharp contrast with LBP $\mathrm{KD}$ cells, that the former were not sensitive to LBP effects, maintaining increased expression levels of adipogenic genes (Fig. $7 \mathrm{c}-\mathrm{g}$ ) and intracellular lipid accumulation (ESM. Fig. 3c), and maintaining a downregulated inflammatory response (such as $I l 6$ gene expression, Fig. $7 \mathrm{~h}$ ). This result suggested the anti-adipogenic effect of LBP requires a functional TLR4 receptor.

\section{Discussion}

Recently, we identified LBP as an adipokine produced in excess by human adipocytes under conditions of positive energy balance, insulin resistance and inflammation in vivo [5]. We here identified that these actions might occur via an enhanced LPS- or palmitate-induced inflammatory response in adipocytes mediated through the TLR4 receptor. These observations are especially relevant given the known LPS and lipid transfer protein activity of LBP [10], but these actions are previously uncharacterised at the adipocyte level.

b

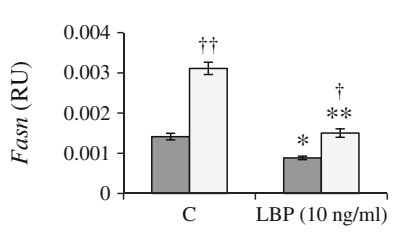

e

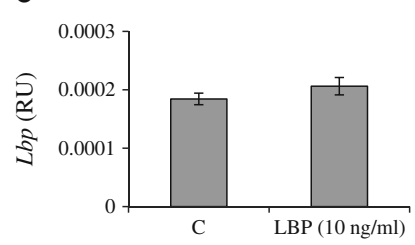

h

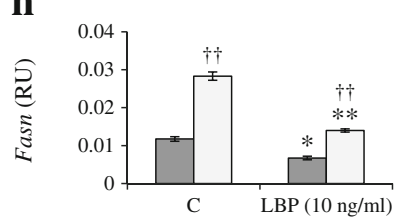

$\mathbf{k}$

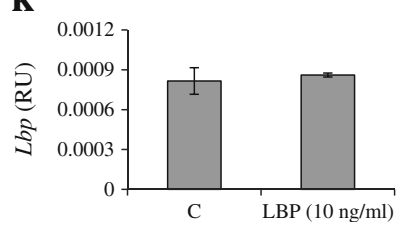

c

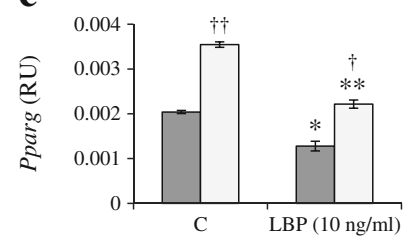

f

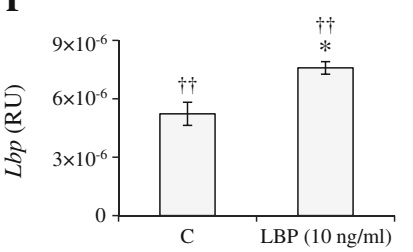

i

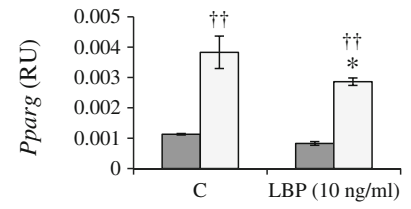

I

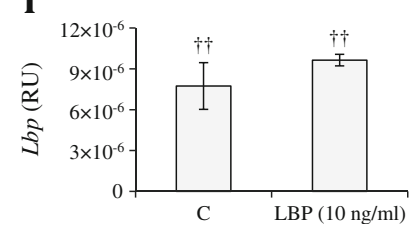


Interestingly, knockdown of LBP led to upregulation of genes linked to adipocyte differentiation and adipocyteassociated metabolic processes, such as mitochondrial biogenesis, citrate cycle, oxidative phosphorylation, fatty acid metabolism and PPAR signalling. Mitochondrial biogenesis and oxidative metabolism are known to be especially important for adipocyte differentiation [11-13] as well as fatty acid metabolism and PPAR signalling [14-16]. Importantly, LBP is also a soluble factor that mediates the effects of inflammatory factors on the adipocyte. LBP silencing led to a dose-related enhancement of 3T3-L1 cell differentiation, which became refractory to the anti-adipogenic effects of inflammatory agents (LPS, NEFA and MCM) and specifically attenuated the decrease in Pparg (the master regulator of adipogenesis), Adipoq (the most specific marker of adipogenesis) and Slc2a 4 (an additional marker of adipogenesis [9]). In agreement with current findings in 3T3-L1 cells, human AT LBP gene expression was significantly associated with obesity-induced AT dysfunction and inflammation, and LBP knockdown (using shRNA lentiviral particles or anti-LBP antibodies) in human adipocytes resulted in increased adipogenic $(A D I P O Q$ and $S L C 2 A 4)$ and decreased IL6 mRNA levels [5].
Chronic inflammation is a crucial pathophysiological factor underlying AT dysfunction [2, 17-19]. The inflammatory resilience of LBP KD cells could be attributed to loss of the LPS- and palmitate-induced inflammatory response. These effects were clearly LBP-dependent because the addition of LBP and co-culture with cells silenced with an empty vector fully reversed these phenotypes, restoring the inflammatory phenotype in LBP KD cells. In agreement with these data, previous studies have shown that LBP contributes, through recognition of the lipid A component of LPS monomers, to accelerate its binding to $\mathrm{CD} 14$, enhancing the sensitivity of cells to LPS [20, 21]. LBP also seems a factor necessary for triggering the inflammatory cascade induced by saturated fatty acids (sFAs) and metabolic endotoxaemia [22-29]. Similar to LPS, saturated fatty acids induce NFKB-mediated adipocyte inflammation through TLR4 [26-29]. As LBP enhances the LPS-TLR4-NFKB pathway [20, 21], we hypothesised that LBP might participate in sFA-induced adipocyte inflammation. Importantly, LBP interacts specifically with lipid A, the hydrophobic anchor of LPS [20]. Supporting this idea and current findings, plasma IL6 and AT markers of inflammation were increased with a palm-oil-enriched diet (rich in
Fig. 5 The co-culture experiment (a). The expression of $\operatorname{Lbp}$ (b), Adipoq (c), Fasn (d), Pparg (e), Cebpa (f) and Mcpl (g) was analysed in si $L b p$ and siC cells and secreted LBP protein was measured in the medium (h) on day $7 .{ }^{*} p<0.05$ and $* * p<0.01$ compared with the siLbp/siLbp system. All experiments were performed in triplicate. Data are represented as mean $\pm \mathrm{SEM}$. RU, relative units

a

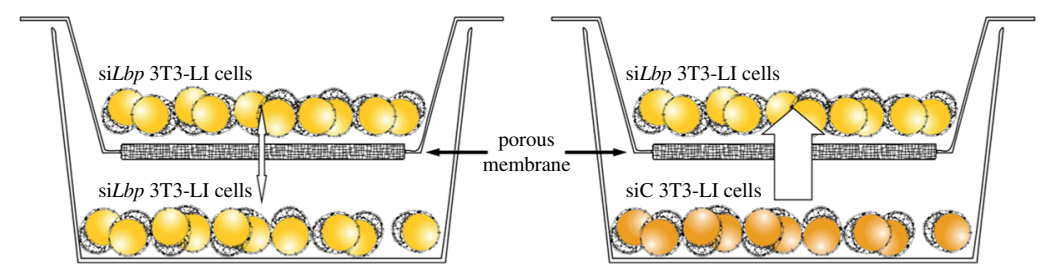

b

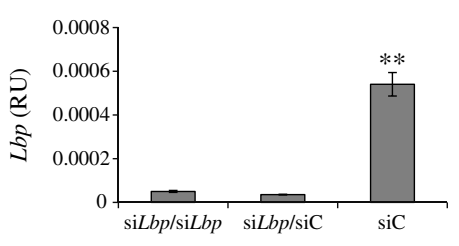

c

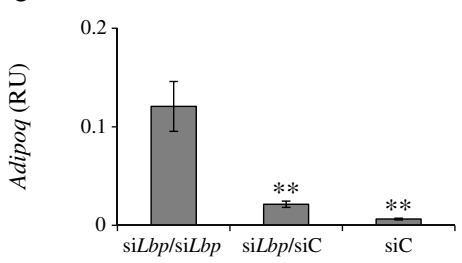

d

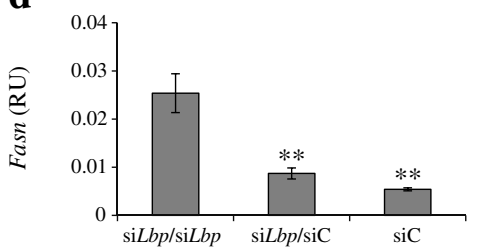

e

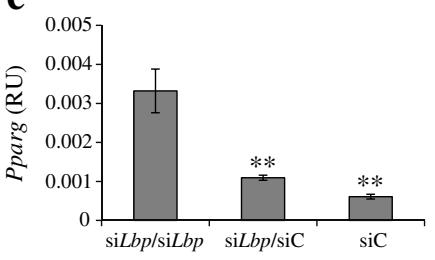

f
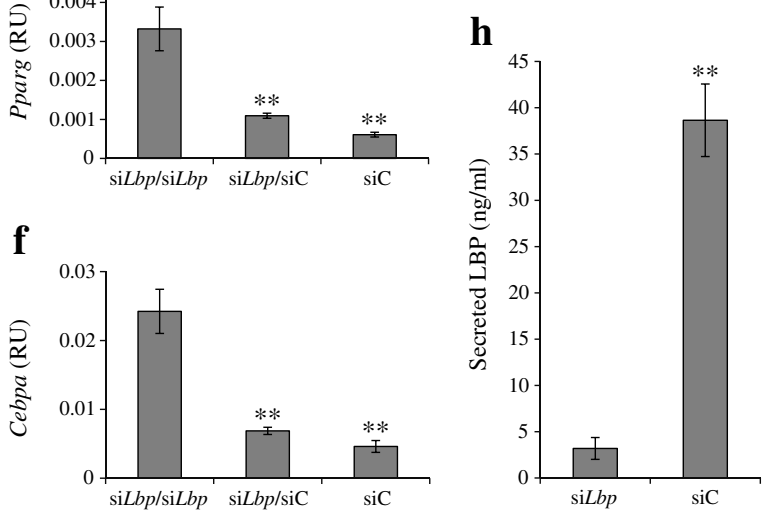

g

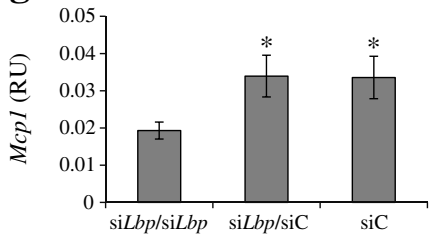


Fig. 6 (a-e) The effects of TLR4 KD in si $L b p$ cells on Tlr4 (a), $L b p$ (b), the adipogenic factors Pparg (c) and Adipoq (d), and Il6 (e) gene expression. All experiments were performed in triplicate. Data are represented as mean $\pm \mathrm{SEM}$. ${ }^{*} p<0.05$ and ${ }^{*} p p<0.01$ compared with day $0 ;{ }^{\dagger} p<0.05$ and ${ }^{\dagger \dagger} p<0.01$ compared with siC; ${ }^{\S_{p}}<0.05$ and ${ }^{\S} p<0.01$ compared with siLbp. Light grey bars, siC; mid grey bars, si $L b p$; and dark grey bars, si $L b p+$ siTlr4. RU, relative units

Fig. 7 A second co-culture experiment used siL $b p$-siTlr43T3-L1 and siC-3T3-L1 cells to test the effects of secreted LBP via the TLR4 pathway. $\operatorname{Lbp}(\mathbf{a})$, Tlr4 (b), Cebpa (c), Pparg (d), Adipoq (e), Fabp4 (f), Slc2a4 (g) and $I l 6(\mathbf{h})$ gene expression at day 7 was measured in each cell group from the co-culture system. The effects of siC-derived conditioned medium on siLbp-siTlr4 (B group) and siC (D group) cells and siLbp-siTlr4-conditioned medium on siLbp-siTlr4 (A group) and $\mathrm{siC}$ (C group) cells were compared. $* * p<0.01$ compared with siLbp-siTlr4/ siLbp-siTlr4 system (A group). ${ }^{\dagger} p<0.05$ compared with $\mathrm{siC} / \mathrm{siC}$ system (D group). All experiments were performed in triplicate. Data are represented as mean \pm SEM. RU, relative units $\mathbf{a}$

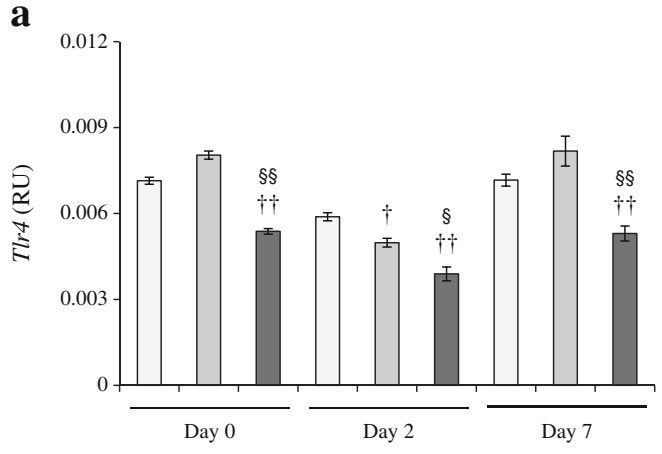

b

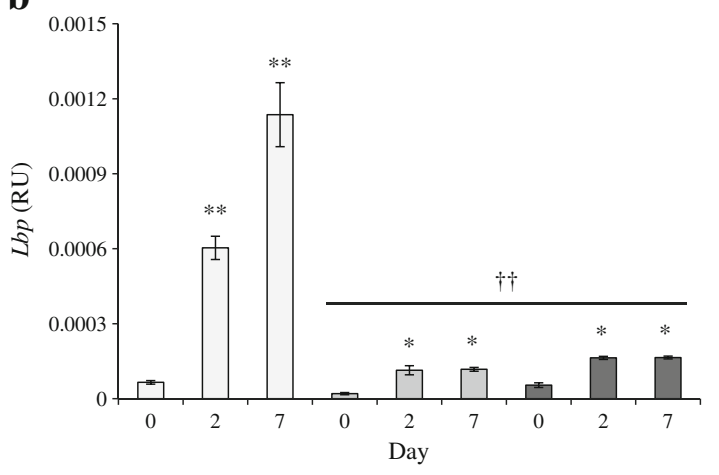

c

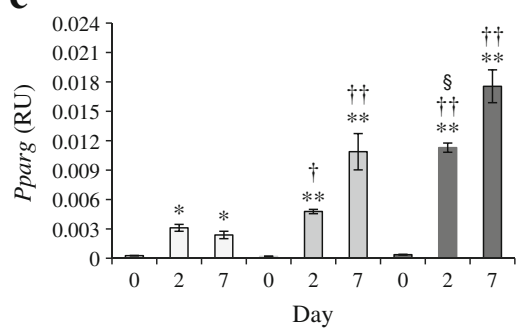

d

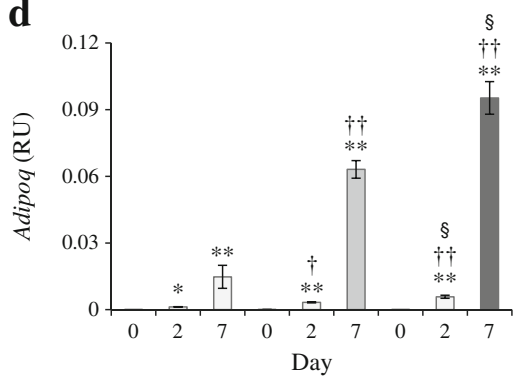

e

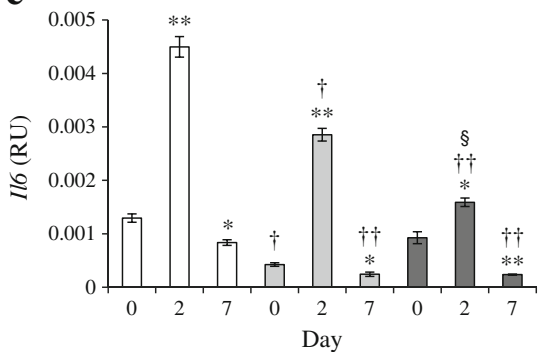

$\mathbf{a}$

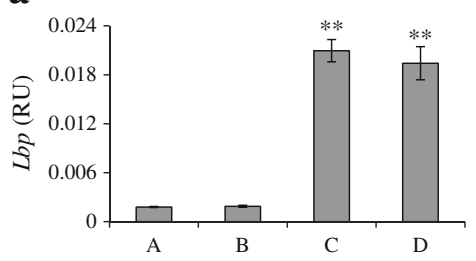

c

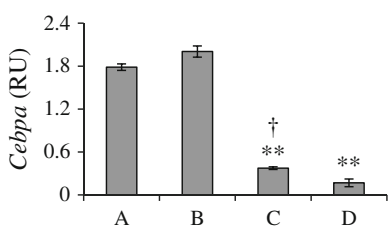

f

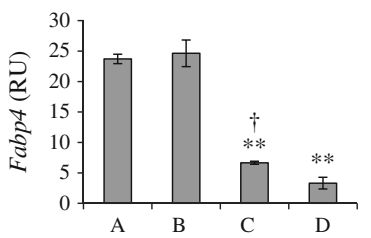

d

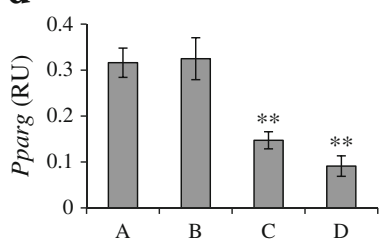

g

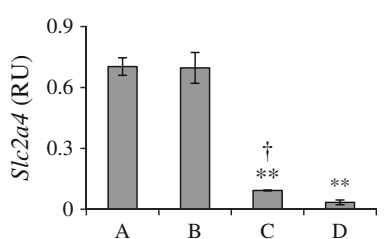

e

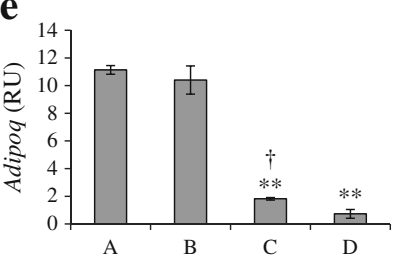

h

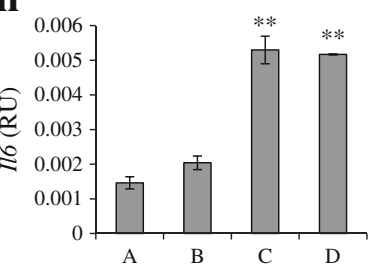


palmitate), and correlated with serum LBP concentration in mice [30]. In fact, as LPS was not detected in cell culture medium, the effects of LBP on adipocyte differentiation possibly depend on the NEFA content of cell culture medium. Activation of ${ }^{\mathrm{p}-\mathrm{S} 536} \mathrm{NFKB}$ under LPS and palmitate stimulation is induced through the TLR4 pathway. The TLR4-LBP $\mathrm{KD}$ cells tended to differentiate better than LBP KD cells (Fig. 7). Again, most of the biological processes and metabolic pathways that changed significantly in TLR4-LBP KD cells vs LBP KD cells related mainly to adipocyte metabolism and adipogenesis (ESM Fig. 2b). Co-culture of TLR4-LBP $\mathrm{KD}$ cells with cells transfected with an empty vector but otherwise able to synthesise LBP (siC-3T3-L1 cells) in a Transwell co-culture system showed that the former were insensitive to LBP effects, maintaining increased expression levels of adipogenic genes and a downregulated inflammatory response. This was in sharp contrast to results with siLbp-3T3L1 cells. All the observations support the hypothesis that the inflammatory effects of soluble LBP require TLR4.

Wernstedt et al have demonstrated that inflammation promotes adipogenesis in vivo and is essential for 'healthy' AT functionality [31]. Adipocyte LBP biosynthesis may contribute to adipogenesis through similar inflammatory pathways. Levels of $L b p$ in epididymal white AT have been shown to parallel the exponential growth phase of AT expansion and adipocyte differentiation in young $o b / o b$ mice [5]. However, additional studies are necessary to confirm the importance of LBP in acute-inflammation-induced AT expandability.

Increased AT lipolysis is associated with insulin resistance and AT dysfunction [32]. It is characterised by a significant increase in the release of sFAs. In this context, increased AT LBP levels might enhance the proinflammatory effects of sFAs on AT, promoting AT dysfunction.

In summary, adipocyte LBP biosynthesis seems to be required to mediate the inflammatory effects induced by positive energy balance, serving as a link between the increased levels of proinflammatory effectors in obesity and their detrimental effects on adipogenesis and adipocyte functionality. Supporting previous in vivo findings $[3,5]$, increased LBP may cause metabolic stress and contribute to a vicious cycle that prevents further AT expansion and exacerbates the inflammatory response in AT. Consequently, LBP constitutes an important target to reverse these effects and restore AT physiology.

Acknowledgements We would like to thank P. Tubert (Laboratori d'Enginyeria de Proteïnes, Department of Biology, Facultat de Ciències, University of Girona, Spain) for help preparing Fig. 5.

Funding This work was partially supported by research grants from the Ministerio de Economía y Competitividad (PI11-00214 and PI12/02631), FONDOS FEDER, European Collaborative Project ADAPT (HEALTHF2-2008-2011 00) and the FLORINASH project. CIBEROBN Fisiopatología de la Obesidad y Nutrición is an initiative from the
Instituto de Salud Carlos III, Spain. AVP is funded by the Medical Research Council (MRC) and Biomedical Research Centre - National Institute for Health Research (BRC-NIHR).

Duality of interest The authors confirm that there is no duality of interest associated with this manuscript.

Contribution statement JMM-N, AZ, AV-P, JV and JMF-R participated in the study design. JMM-N, XE, FO, MC, WR, AZ, AV-P, JV and JMF-R participated in the acquisition, analysis and interpretation of data and drafting the article or revising it critically for important intellectual content. All authors gave final approval of the version to be published. JMM-N and JMF-R wrote and edited the manuscript and are the guarantors of this work.

\section{References}

1. Wormser D, Kaptoge S, Di Angelantonio E et al (2011) Separate and combined associations of body-mass index and abdominal adiposity with cardiovascular disease: collaborative analysis of 58 prospective studies. Lancet 377:1085-1095

2. Ouchi N, Parker JL, Lugus JJ et al (2011) Adipokines in inflammation and metabolic disease. Nat Rev Immunol 11:85-97

3. Ge Q, Gérard J, Noël L et al (2012) MicroRNAs regulated by adiponectin as novel targets for controlling adipose tissue inflammation. Endocrinology 153:5285-5296

4. Nakarai H, Yamashita A, Nagayasu S et al (2012) Adipocytemacrophage interaction may mediate LPS-induced low-grade inflammation: potential link with metabolic complications. Innate Immun 18:164-170

5. Moreno-Navarrete JM, Escoté X, Ortega F et al (2013) A role for adipocyte derived lipopolysaccharide binding protein in inflammation- and obesity-associated adipose tissue dysfunction. Diabetologia 56:2524-2537

6. Geurts L, Everard A, Van Hul M et al (2015) Adipose tissue NAPEPLD controls fat mass development by altering the browning process and gut microbiota. Nat Commun 6:6495

7. Guo W, Wong S, Xie W, Lei T, Luo Z (2007) Palmitate modulates intracellular signaling, induces endoplasmic reticulum stress, and causes apoptosis in mouse 3T3-L1 and rat primary preadipocytes. Am J Physiol Endocrinol Metab 293:E576-E586

8. Wang Y, Kim KA, Kim JH, Sul HS (2006) Pref-1, a preadipocyte secreted factor that inhibits adipogenesis. J Nutr 136:2953-2956

9. Cha HC, Oak NR, Kang S et al (2008) Phosphorylation of CCAAT/ enhancer-binding protein alpha regulates GLUT4 expression and glucose transport in adipocytes. J Biol Chem 283:18002-18011

10. Kirschning CJ, Au-Young J, Lamping N et al (1997) Similar organization of the lipopolysaccharide-binding protein (LBP) and phospholipid transfer protein (PLTP) genes suggests a common gene family of lipid-binding proteins. Genomics 46:416-425

11. Huh JY, Kim Y, Jeong J et al (2012) Peroxiredoxin 3 is a key molecule regulating adipocyte oxidative stress, mitochondrial biogenesis, and adipokine expression. Antioxid Redox Signal 16:229-243

12. Hofmann AD, Beyer M, Krause-Buchholz U et al (2012) OXPHOS supercomplexes as a hallmark of the mitochondrial phenotype of adipogenic differentiated human MSCs. PLoS One 7:e35160

13. Ducluzeau PH, Priou M, Weitheimer M et al (2011) Dynamic regulation of mitochondrial network and oxidative functions during 3T3-L1 fat cell differentiation. J Physiol Biochem 67:285-296 
14. Greenberg AS, Coleman RA, Kraemer FB et al (2011) The role of lipid droplets in metabolic disease in rodents and humans. J Clin Invest 121:2102-2110

15. Vigouroux C, Caron-Debarle M, Le Dour C et al (2011) Molecular mechanisms of human lipodystrophies: from adipocyte lipid droplet to oxidative stress and lipotoxicity. Int J Biochem Cell Biol 43:862-876

16. Cristancho AG, Lazar MA (2011) Forming functional fat: a growing understanding of adipocyte differentiation. Nat Rev Mol Cell Biol $12: 722-734$

17. Vitseva OI, Tanriverdi K, Tchkonia TT et al (2008) Inducible tolllike receptor and NF- $\mathrm{KB}$ regulatory pathway expression in human adipose tissue. Obesity (Silver Spring) 16:932-937

18. Gillum MP, Kotas ME, Erion DM et al (2011) SirT1 regulates adipose tissue inflammation. Diabetes 60:3235-3245

19. Lê KA, Mahurkar S, Alderete TL et al (2011) Subcutaneous adipose tissue macrophage infiltration is associated with hepatic and visceral fat deposition, hyperinsulinemia, and stimulation of NF- $\mathrm{kB}$ stress pathway. Diabetes 60:2802-2809

20. Tobias PS, Soldau K, Ulevitch RJ (1989) Identification of a lipid A binding site in the acute phase reactant lipopolysaccharide binding protein. J Biol Chem 264:10867-10871

21. Hailman E, Lichenstein HS, Wurfel MM et al (1994) Lipopolysaccharide (LPS)-binding protein accelerates the binding of LPS to CD14. J Exp Med 179:269-277

22. Cani PD, Amar J, Iglesias MA et al (2007) Metabolic endotoxemia initiates obesity and insulin resistance. Diabetes 56:1761-1772

23. Osto M, Zini E, Franchini M et al (2011) Subacute endotoxemia induces adipose inflammation and changes in lipid and lipoprotein metabolism in cats. Endocrinology 152:804-815

24. Chung S, Lapoint K, Martinez K et al (2006) Preadipocytes mediate lipopolysaccharide-induced inflammation and insulin resistance in primary cultures of newly differentiated human adipocytes. Endocrinology 147:5340-5351

25. Creely SJ, McTernan PG, Kusminski CM et al (2007) Lipopolysaccharide activates an innate immune system response in human adipose tissue in obesity and type 2 diabetes. Am J Physiol Endocrinol Metab 292:E740-E747

26. Suganami T, Tanimoto-Koyama K, Nishida J et al (2007) Role of the Toll-like receptor $4 / \mathrm{NF}-\mathrm{KB}$ pathway in saturated fatty acidinduced inflammatory changes in the interaction between adipocytes and macrophages. Arterioscler Thromb Vasc Biol 27:84-91

27. Huang S, Rutkowsky JM, Snodgrass RG et al (2012) Saturated fatty acids activate TLR-mediated proinflammatory signaling pathways. J Lipid Res 53:2002-2013

28. Shi H, Kokoeva MV, Inouye K et al (2006) TLR4 links innate immunity and fatty acid-induced insulin resistance. J Clin Invest 116:3015-3025

29. Youssef-Elabd EM, McGee KC, Tripathi G et al (2012) Acute and chronic saturated fatty acid treatment as a key instigator of the TLRmediated inflammatory response in human adipose tissue, in vitro. J Nutr Biochem 23:39-50

30. Laugerette F, Furet JP, Debard C et al (2012) Oil composition of high-fat diet affects metabolic inflammation differently in connection with endotoxin receptors in mice. Am J Physiol Endocrinol Metab 302:E374-E386

31. Wernstedt Asterholm I, Tao C, Morley TS et al (2014) Adipocyte inflammation is essential for healthy adipose tissue expansion and remodeling. Cell Metab 20:103-118

32. Girousse A, Tavernier G, Valle C et al (2013) Partial inhibition of adipose tissue lipolysis improves glucose metabolism and insulin sensitivity without alteration of fat mass. PLoS Biol 11:e1001485 